\title{
Communication \\ More Than Meets the Kappa for Antibody Superantigen Protein L (PpL)
}

\author{
Wei-Li Ling ${ }^{1,2}$, Joshua Yi Yeo ${ }^{1}\left(\mathbb{D}\right.$, Yuen-Ling $\mathrm{Ng}^{2}$, Anil Wipat ${ }^{3}$ and Samuel Ken-En Gan ${ }^{1,4, *(\mathbb{D})}$ \\ 1 Antibody \& Product Development Laboratory, Experimental Drug Development Centre-Bioinformatics \\ Institute, Agency for Science, Technology and Research (A*STAR), Singapore 138672, Singapore; \\ alexling1989@gmail.com (W.-L.L.); joshy@live.com.sg (J.Y.Y.) \\ 2 Newcastle Research and Innovation Institute (NewRIIS), Singapore 609607, Singapore; \\ yuenling.ng@newcastle.ac.uk \\ 3 School of Computing, Newcastle University, Newcastle upon Tyne NE1 7RU, UK; anil.wipat@newcastle.ac.uk \\ 4 James Cook University, Singapore 387380, Singapore \\ * Correspondence: samgan@apdskeg.com
}

check for updates

Citation: Ling, W.-L.; Yeo, J.Y.; Ng, Y.-L.; Wipat, A.; Gan, S.K.-E. More Than Meets the Kappa for Antibody Superantigen Protein L

(PpL). Antibodies 2022, 11, 14.

https://doi.org/10.3390/

antib11010014

Received: 5 January 2022

Accepted: 7 February 2022

Published: 11 February 2022

Publisher's Note: MDPI stays neutral with regard to jurisdictional claims in published maps and institutional affiliations.

Copyright: (C) 2022 by the authors. Licensee MDPI, Basel, Switzerland. This article is an open access article distributed under the terms and conditions of the Creative Commons Attribution (CC BY) license (https:// creativecommons.org/licenses/by/ $4.0 /)$.

\begin{abstract}
Immunoglobulin superantigens play an important role in affinity purification of antibodies and the microbiota-immune axis at mucosal areas. Based on current understanding, Staphylococcal Protein A (SpA), Streptococcal Protein G (SpG) and Finegoldia Protein L (PpL) are thought to only bind specific regions of human antibodies, allowing for selective purification of antibody isotypes and chains. Clinically, these superantigens are often classified as toxins and increase the virulence of the producing pathogen through unspecific interactions with immune proteins. To perform an in-depth interaction study of these three superantigens with antibodies, bio-layer interferometry (BLI) measurements of their interactions with a permutation panel of 63 IgG1 variants of Pertuzumab and Trastuzumab CDRs grafted to the six human VK and seven human VH region families were tested. Through this holistic and systemic analysis of IgG1 variants with various antibody regions modified, comparisons revealed novel PpL-antibody interactions influenced by other non-canonical antibody known light-chain framework regions, whereas SpA and SpG showed relatively consistent interactions. These findings have implications on PpL-based affinity antibody purification and design that can guide the engineering and understanding of PpL-based microbiota-immune effects.
\end{abstract}

Keywords: Pertuzumab; Trastuzumab; IgG1; VH families; VK families; immunoglobulin; superantigen

\section{Introduction}

B cell superantigens bind antibodies to hyperstimulate populations of B cells independent of $\mathrm{T}$ cells and have been widely used for antibody affinity purification [1]. Superantigens are predominantly produced by microorganisms as a defence mechanism to escape the host immune system [2]. Notably, there are three widely used antibody superantigens also known as immunoglobulin binding proteins (IBP): Protein G (SpG) produced by groups C and G of Streptococcal bacteria [3], which binds to the heavy chain constant region of the IgG subtypes (IgG1-4); Protein A (SpA) produced by Staphylococcus aureus, which binds to the heavy chain constant region of $\operatorname{IgG1}, 2$, and 4 and the variable heavy (VH) 3 framework (VH3) [4-6]; and Protein L (PpL) produced by Finegoldia magna (previously known as Peptostreptococcus magnus), which binds to the variable light kappa $\mathrm{K}$ (Vk) chain families 1,3,4 [7] at the framework (FWR) 1 region with influence from the other regions, e.g., distal FWRs [8]. When bound to antibodies, these superantigens can reduce the binding of the antibodies to their target antigens [9], possibly reducing avidity through steric hindrances as in the case of IgM [10]. They can also cause unwanted activation [4] with downstream effects depending on their target antibody isotype (discussed in [11,12]).

With both IgG and Vk being the predominant isotypes in humans [13,14], superantigen proteins $A, G$, and $L$ can underlie significant microbiota-immune axis interactions 
especially at colonised mucosal areas. Many therapeutic antibodies of the IgG and $\mathrm{k}$ light chain isotypes, most notably the well-studied Pertuzumab and Trastuzumab antibodies which target Her2-positive breast cancer. 30\% of invasive breast cancers and $70 \%$ of ductal carcinomas were found to overexpress Her2 antigen [15,16], making it an attractive target for anti-cancer therapy. Trastuzumab (Herceptin ${ }^{\circledR}$ ), a humanised monoclonal IgG1 antibody with its V-region derived from rodents [17], is often coupled with Pertuzumab (Perjeta ${ }^{\circledR}$ ) [18] in clinical therapy. Both Trastuzumab and Pertuzumab can co-localize on Her2 as they bind at different sites on Her2 [19] and have been shown to effectively treat Her2-overexpressing cancers synergistically. Using these two antibody models, we investigated unwanted interactions of such superantigens produced by commensals. In addition, implications of these antibody-superantigen interactions extend to antibody purification processes which often utilises these superantigens.

Given the common use of these three superantigens, a holistic [12,20] and systematic antibody-superantigen investigation using 63 of our previously engineered antibodies [21-23] involving the permutations of six human $\mathrm{Vk}$ and seven human $\mathrm{VH}$ IgGs was performed. These recombinant antibodies were engineered based on the complementarity determining regions (CDR)-grafting of the Trastuzumab and Pertuzumab antibodies onto the human antibody FWRs on both heavy and light chains, allowing for a systematic analysis of these regions (V-region, VH, Vk, FWRs, and CDRs of both chains, and the synergistic interactions) in superantigen engagements.

\section{Materials and Methods}

\subsection{Recombinant Antibody Production}

All Trastuzumab and Pertuzumab VH and Vk CDR-grafted sequences used were described previously [21,22]. Briefly, the genes were sub-cloned into the pTT5 vector (Youbio, Changsha, China, Cat: VT2202) using restriction enzyme sites as previously performed [8,21-24]. The plasmids were transformed into competent E. coli (DH5 $\alpha)$ strains [25] followed by plasmid extraction (Biobasic Pte Ltd, Singapore, Singapore, Cat: BS614). Transfection, production, and purification were done using HEK293E with homemade transfection agent based on linear PEI [26] (PolyScience, Singpaore, Singapore, Cat: 23966-1), followed by 14 days post-transfection supernatant harvest that were subjected to AKTA Pure system equipped with Protein G column for affinity purification and size exclusion column for collection of monomeric fractions as characterised previously [21,22].

\subsection{Binding Affinity Quantification}

Measurements of the rates of association $(\mathrm{ka})$ and rates of dissociation $(\mathrm{kd})$ were performed using PpL (Sartorius, Singapore, Singapore, Cat: 18-5185), SpA (Sartorius, Singapore, Cat: 18-5012), and SpG (Sartorius, Singapore, Cat: 18-18-5083) biosensors to Pertuzumab and Trastuzumab IgG1 variants described above in solution using the Octet Red $96^{\circledR}$ system. The Octet Acquisition v10.0 program was used to set and test the respective biosensors with the following steps for measurements: Pre-conditioning (0.2 M glycine, pH 1.52 \& $10 \times$ kinetic buffer (KB), 30 s); Initial Baseline $(10 \times \mathrm{KB}, 60 \mathrm{~s})$; Baseline $(10 \times \mathrm{KB}$, $120 \mathrm{~s})$; Association (antibody variants, $6.25 \mathrm{nM}-100 \mathrm{nM}, 300 \mathrm{~s}) ;$ Dissociation $(10 \times \mathrm{KB}$, 600 s); Regeneration (0.2 M glycine, pH 1.52 \& 10× kinetic buffer (KB), 30 s) as previously described $[4,8,21-24,27]$. The equilibrium dissociation constant (KD), ka and kd were automatically calculated by the Data Analysis v10.0 program using a 1:1 fitting model. KD measurements were deemed reliable if the response rates were above 0.1 of the respective concentrations (6.25 $\mathrm{nM}-100 \mathrm{nM})$ as recommended by the manufacturer.

\section{Results}

\subsection{BLI Measurement of Recombinant IgG1 Variants to Protein A (SpA)}

To examine the potential holistic effects of Vk1-6 pairing with VH1-7 on antibody interactions with SpA, recombinant Pertuzumab and Trastuzumab IgG1 variants of the 
various pairings were studied. Notably, $\mathrm{SpA}$ is known to bind to the $\mathrm{CH} 2$ and $\mathrm{CH} 3$ domains of the heavy chain constant $(\mathrm{CH})$.

From Figure 1, the Pertuzumab and Trastuzumab IgG1 variants showed measurements bound to SpA with the KD at 0.41-1.90 $\times 10^{-9} \mathrm{M}$ and $0.24-2.30 \times 10^{-9} \mathrm{M}$, respectively (refer to Supplementary Figures S1-S4 for the graphs).

\begin{tabular}{|c|c|c|c|c|c|c|}
\hline \multirow{2}{*}{$\begin{array}{l}\text { Vк-VH } \\
\text { Pairing }\end{array}$} & \multicolumn{3}{|c|}{ Pertuzumab variants binding $\mathrm{SpA}$} & \multicolumn{3}{|c|}{ Trastuzumab variants binding SpA } \\
\hline & $\begin{array}{c}\text { KD } \\
\left(M, 10^{-9}\right)\end{array}$ & $\begin{array}{c}\text { ka } \\
\left(1 / \mathrm{Ms}, 10^{4}\right)\end{array}$ & $\begin{array}{c}\text { kd } \\
\left(1 / \mathrm{s}, 10^{-4}\right)\end{array}$ & $\begin{array}{c}\text { KD } \\
\left(M, 10^{-9}\right)\end{array}$ & $\begin{array}{c}\text { ka } \\
\left(1 / \mathrm{Ms}, 10^{4}\right)\end{array}$ & $\begin{array}{c}\text { kd } \\
\left(1 / \mathrm{s}, 10^{-4}\right)\end{array}$ \\
\hline$\kappa 1-\mathrm{H1}$ & $1.58 \pm 0.01$ & $37.79 \pm 0.26$ & $5.92 \pm 0.03$ & \multirow{4}{*}{\multicolumn{3}{|c|}{ Not Produced }} \\
\hline$\kappa 1-\mathrm{H} 2$ & $1.58 \pm 0.01$ & $41.43 \pm 0.28$ & $6.56 \pm 0.04$ & & & \\
\hline$\kappa 1-\mathrm{H} 3$ & $0.64 \pm 0.01$ & $53.29 \pm 0.32$ & $3.26 \pm 0.03$ & & & \\
\hline$\kappa 1-\mathrm{H} 4$ & $1.56 \pm 0.01$ & $38.64 \pm 0.26$ & $6.01 \pm 0.03$ & & & \\
\hline к1-H5 & \multirow{2}{*}{\multicolumn{3}{|c|}{ Not Produced }} & $1.06 \pm 0.02$ & $29.07 \pm 0.24$ & $3.07 \pm 0.04$ \\
\hline к1-H6 & & & & \multicolumn{3}{|c|}{ Not Produced } \\
\hline к1-H7 & $1.17 \pm 0.01$ & $42.74 \pm 0.27$ & $5.02 \pm 0.03$ & $0.66 \pm 0.01$ & $44.31 \pm 0.19$ & $2.93 \pm 0.02$ \\
\hline$\kappa 2-\mathrm{H1}$ & $1.90 \pm 0.02$ & $33.08 \pm 0.22$ & $6.32 \pm 0.03$ & $1.49 \pm 0.01$ & $17.97 \pm 0.08$ & $2.68 \pm 0.02$ \\
\hline$\kappa 2-\mathrm{H} 2$ & $1.82 \pm 0.02$ & $35.57 \pm 0.24$ & $6.47 \pm 0.04$ & $1.46 \pm 0.02$ & $17.32 \pm 0.06$ & $2.14 \pm 0.02$ \\
\hline$\kappa 2-\mathrm{H} 3$ & $0.76 \pm 0.01$ & $55.68 \pm 0.34$ & $4.23 \pm 0.03$ & $0.24 \pm 0.01$ & $45.66 \pm 0.21$ & $1.08 \pm 0.02$ \\
\hline$\kappa 2-\mathrm{H} 4$ & $1.57 \pm 0.01$ & $36.21 \pm 0.22$ & $5.68 \pm 0.03$ & $1.29 \pm 0.01$ & $35.58 \pm 0.27$ & $4.51 \pm 0.04$ \\
\hline$\kappa 2-\mathrm{H5}$ & $1.62 \pm 0.01$ & $35.83 \pm 0.22$ & $5.82 \pm 0.03$ & $1.11 \pm 0.01$ & $60.04 \pm 0.44$ & $6.64 \pm 0.04$ \\
\hline$\kappa 2-\mathrm{H} 6$ & $1.55 \pm 0.01$ & $38.00 \pm 0.23$ & $5.91 \pm 0.03$ & $1.79 \pm 0.02$ & $25.36 \pm 0.15$ & $4.51 \pm 0.03$ \\
\hline$\kappa 2-\mathrm{H} 7$ & $1.15 \pm 0.01$ & $47.37 \pm 0.31$ & $5.44 \pm 0.03$ & $0.53 \pm 0.01$ & $57.16 \pm 0.28$ & $3.04 \pm 0.02$ \\
\hline$\kappa 3-\mathrm{H1}$ & $1.06 \pm 0.01$ & $37.25 \pm 0.19$ & $3.92 \pm 0.03$ & $0.83 \pm 0.01$ & $40.56 \pm 0.17$ & $3.35 \pm 0.02$ \\
\hline$\kappa 3-\mathrm{H} 2$ & $1.57 \pm 0.01$ & $40.11 \pm 0.26$ & $6.30 \pm 0.03$ & $1.11 \pm 0.01$ & $32.64 \pm 0.15$ & $3.60 \pm 0.02$ \\
\hline$\kappa 3-\mathrm{H3}$ & $0.69 \pm 0.01$ & $58.94 \pm 0.32$ & $4.07 \pm 0.03$ & $0.26 \pm 0.01$ & $41.54 \pm 0.18$ & $1.06 \pm 0.02$ \\
\hline$\kappa 3-\mathrm{H} 4$ & $1.50 \pm 0.01$ & $36.19 \pm 0.24$ & $5.45 \pm 0.03$ & $2.14 \pm 0.03$ & $14.09 \pm 0.09$ & $3.01 \pm 0.03$ \\
\hline$\kappa 3-\mathrm{H5}$ & $1.57 \pm 0.01$ & $39.18 \pm 0.25$ & $6.18 \pm 0.03$ & $0.73 \pm 0.01$ & $24.45 \pm 0.13$ & $1.78 \pm 0.03$ \\
\hline$\kappa 3-\mathrm{H} 6$ & $1.43 \pm 0.01$ & $42.28 \pm 0.27$ & $6.04 \pm 0.03$ & $1.61 \pm 0.02$ & $38.54 \pm 0.28$ & $6.15 \pm 0.04$ \\
\hline к3-Н7 & $1.12 \pm 0.01$ & $44.12 \pm 0.26$ & $4.93 \pm 0.03$ & $0.56 \pm 0.01$ & $46.35 \pm 0.22$ & $2.60 \pm 0.02$ \\
\hline$\kappa 4-\mathrm{H1}$ & $1.70 \pm 0.01$ & $37.79 \pm 0.24$ & $6.43 \pm 0.03$ & $1.95 \pm 0.02$ & $18.22 \pm 0.11$ & $3.55 \pm 0.03$ \\
\hline$\kappa 4-\mathrm{H} 2$ & $1.46 \pm 0.01$ & $39.39 \pm 0.25$ & $5.77 \pm 0.03$ & \multicolumn{3}{|c|}{ Not Produced } \\
\hline$\kappa 4-\mathrm{H} 3$ & $0.58 \pm 0.01$ & $43.12 \pm 0.31$ & $2.46 \pm 0.03$ & $0.25 \pm 0.01$ & $45.00 \pm 0.21$ & $1.08 \pm 0.02$ \\
\hline$\kappa 4-\mathrm{H} 4$ & $1.61 \pm 0.01$ & $36.02 \pm 0.24$ & $5.73 \pm 0.03$ & $1.67 \pm 0.02$ & $32.31 \pm 0.21$ & $5.36 \pm 0.03$ \\
\hline к4-H5 & $1.88 \pm 0.02$ & $34.13 \pm 0.22$ & $6.40 \pm 0.03$ & $1.12 \pm 0.02$ & $20.69 \pm 0.17$ & $2.31 \pm 0.04$ \\
\hline$\kappa 4-\mathrm{H} 6$ & $1.85 \pm 0.01$ & $31.10 \pm 0.18$ & $5.76 \pm 0.03$ & $2.30 \pm 0.02$ & $23.37 \pm 0.14$ & $5.33 \pm 0.03$ \\
\hline$\kappa 4-\mathrm{H} 7$ & $1.33 \pm 0.01$ & $36.54 \pm 0.20$ & $4.85 \pm 0.03$ & $0.53 \pm 0.01$ & $64.07 \pm 0.34$ & $3.25 \pm 0.02$ \\
\hline$\kappa 5-\mathrm{H1}$ & \multirow{2}{*}{\multicolumn{3}{|c|}{ Not Produced }} & \multirow{2}{*}{\multicolumn{3}{|c|}{ Not Produced }} \\
\hline$\kappa 5-\mathrm{H} 2$ & & & & & & \\
\hline$\kappa 5-\mathrm{H} 3$ & $0.94 \pm 0.02$ & $18.65 \pm 0.10$ & $1.74 \pm 0.03$ & $0.72 \pm 0.01$ & $24.60 \pm 0.11$ & $1.74 \pm 0.02$ \\
\hline к5-H4 & \multirow{3}{*}{\multicolumn{3}{|c|}{ Not Produced }} & \multirow{4}{*}{\multicolumn{3}{|c|}{ Not Produced }} \\
\hline$\kappa 5-\mathrm{H} 5$ & & & & & & \\
\hline$\kappa 5-\mathrm{H} 6$ & & & & & & \\
\hline к5-H7 & $1.30 \pm 0.01$ & $34.41 \pm 0.19$ & $4.49 \pm 0.03$ & & & \\
\hline к6-H1 & $0.94 \pm 0.01$ & $33.10 \pm 0.16$ & $3.29 \pm 0.02$ & $0.57 \pm 0.01$ & $27.22 \pm 0.09$ & $1.52 \pm 0.02$ \\
\hline$\kappa 6-\mathrm{H} 2$ & $0.79 \pm 0.01$ & $36.64 \pm 0.17$ & $2.93 \pm 0.02$ & \multicolumn{3}{|c|}{ Not Produced } \\
\hline$\kappa 6-\mathrm{H3}$ & $0.41 \pm 0.01$ & $47.42 \pm 0.21$ & $2.12 \pm 0.02$ & $0.31 \pm 0.01$ & $42.43 \pm 0.18$ & $1.31 \pm 0.02$ \\
\hline$\kappa 6-\mathrm{H} 4$ & $0.81 \pm 0.01$ & $36.58 \pm 0.18$ & $2.64 \pm 0.03$ & $1.80 \pm 0.02$ & $21.44 \pm 0.11$ & $3.86 \pm 0.03$ \\
\hline$\kappa 6-\mathrm{H5}$ & $1.26 \pm 0.01$ & $46.77 \pm 0.30$ & $5.85 \pm 0.03$ & $0.84 \pm 0.01$ & $83.03 \pm 0.71$ & $6.97 \pm 0.04$ \\
\hline к6-Н6 & \multicolumn{3}{|c|}{ Not Produced } & $1.78 \pm 0.02$ & $34.99 \pm 0.29$ & $6.15 \pm 0.04$ \\
\hline$\kappa 6-\mathrm{H} 7$ & $0.98 \pm 0.01$ & $49.74 \pm 0.30$ & $4.85 \pm 0.03$ & $0.60 \pm 0.01$ & $50.44 \pm 0.25$ & $2.98 \pm 0.02$ \\
\hline
\end{tabular}

Figure 1. BLI measurements (KD, ka, and kd) of Pertuzumab and Trastuzumab Vk1-6 and VH17variants binding to the immobilised SpA biosensor. "Not Produced" denotes insufficient antibody production for the variant despite numerous large-scale transfections. All readings were obtained from at least three antibody concentrations. The readings were the average of independent triplicates. The graphs are shown in Supplementary Figures S1-S4.

For both Pertuzumab and Trastuzumab variants binding to SpA, VH3 was noticed to have a slightly lower, albeit insignificant KD difference at the average of $\sim 0.67 \times 10^{-9} \mathrm{M}$ and $\sim 0.36 \times 10^{-9} \mathrm{M}$, respectively, especially when compared to the other $\mathrm{VH}$ families paired with the same $\mathrm{V} \kappa$ family. This phenomenon is attributed to the higher ka and lower $\mathrm{kd}$ for the VH3 variant. 


\subsection{BLI Measurement of Recombinant IgG1 Variants to Protein G (SpG)}

Testing the 63 recombinant Pertuzumab and Trastuzumab IgG1 variants with SpG (Figure 2), known to bind the $\mathrm{CH} 2$ and $\mathrm{CH} 3$ domains, we found high consistency of the interactions between the two Pertuzumab and Trastuzumab IgG1 variants. Apart from showing similar KDs to the SpA, SpG interactions showed narrower KD ranges of $0.23-0.87 \times 10^{-9} \mathrm{M}$ and $0.22-0.69 \times 10^{-9} \mathrm{M}$, respectively (refer to Supplementary Figures S5-S8 for the graphs). A trend of Trastuzumab variants binding SpG better than their Pertuzumab counterparts was observed and this slight difference hints of complementaritydetermining regions (CDRs) effects given that the Pertuzumab and Trastuzumab variants differed only at a few residues within the CDRs.

\begin{tabular}{|c|c|c|c|c|c|c|}
\hline \multirow{2}{*}{$\begin{array}{l}\text { Vк-VН } \\
\text { Pairing }\end{array}$} & \multicolumn{3}{|c|}{ Pertuzumab variants binding SpG } & \multicolumn{3}{|c|}{ Trastuzumab variants binding $\mathrm{SpG}$} \\
\hline & $\begin{array}{c}\text { KD } \\
\left(\mathrm{M}, 10^{-9}\right)\end{array}$ & $\begin{array}{c}\text { ka } \\
\left(1 / \mathrm{Ms}, 10^{4}\right)\end{array}$ & $\begin{array}{c}\text { kd } \\
\left(1 / \mathrm{s}, 10^{-4}\right)\end{array}$ & $\begin{array}{c}\text { KD } \\
\left(\mathrm{M}, 10^{-9}\right)\end{array}$ & $\begin{array}{c}\text { ka } \\
\left(1 / \mathrm{Ms}, 10^{4}\right)\end{array}$ & $\begin{array}{c}\text { kd } \\
\left(1 / s, 10^{-4}\right)\end{array}$ \\
\hline к1-H1 & $0.28 \pm 0.01$ & $33.22 \pm 0.13$ & $0.92 \pm 0.03$ & \multirow{4}{*}{\multicolumn{3}{|c|}{ Not Produced }} \\
\hline к1-H2 & $0.31 \pm 0.01$ & $32.59 \pm 0.12$ & $1.02 \pm 0.03$ & & & \\
\hline к1-H3 & $0.87 \pm 0.02$ & $30.74 \pm 0.14$ & $2.22 \pm 0.04$ & & & \\
\hline к1-H4 & $0.30 \pm 0.01$ & $35.54 \pm 0.14$ & $1.04 \pm 0.03$ & & & \\
\hline к1-H5 & \multirow{2}{*}{\multicolumn{3}{|c|}{ Not Produced }} & $0.29 \pm 0.01$ & $32.31 \pm 0.12$ & $0.91 \pm 0.03$ \\
\hline к1-H6 & & & & \multicolumn{3}{|c|}{ Not Produced } \\
\hline к1-H7 & $0.38 \pm 0.01$ & $29.75 \pm 0.11$ & $1.12 \pm 0.03$ & $0.39 \pm 0.01$ & $30.19 \pm 0.10$ & $1.16 \pm 0.02$ \\
\hline$\kappa 2-\mathrm{H} 1$ & $0.33 \pm 0.01$ & $29.12 \pm 0.11$ & $0.96 \pm 0.03$ & $0.39 \pm 0.01$ & $25.84 \pm 0.09$ & $1.00 \pm 0.02$ \\
\hline$\kappa 2-\mathrm{H} 2$ & $0.36 \pm 0.01$ & $29.18 \pm 0.11$ & $1.05 \pm 0.03$ & $0.69 \pm 0.01$ & $22.85 \pm 0.05$ & $1.57 \pm 0.03$ \\
\hline$\kappa 2-\mathrm{H} 3$ & $0.38 \pm 0.01$ & $31.21 \pm 0.11$ & $1.16 \pm 0.03$ & $0.29 \pm 0.01$ & $34.26 \pm 0.13$ & $0.98 \pm 0.03$ \\
\hline$\kappa 2-\mathrm{H} 4$ & $0.25 \pm 0.01$ & $34.56 \pm 0.14$ & $0.83 \pm 0.03$ & $0.28 \pm 0.01$ & $43.48 \pm 0.17$ & $1.15 \pm 0.03$ \\
\hline$\kappa 2-\mathrm{H5}$ & $0.30 \pm 0.01$ & $28.66 \pm 0.11$ & $0.86 \pm 0.03$ & $0.27 \pm 0.01$ & $37.63 \pm 0.14$ & $0.97 \pm 0.03$ \\
\hline к2-H6 & $0.32 \pm 0.01$ & $30.00 \pm 0.11$ & $0.95 \pm 0.03$ & $0.33 \pm 0.01$ & $25.29 \pm 0.09$ & $0.84 \pm 0.02$ \\
\hline$\kappa 2-\mathrm{H} 7$ & $0.36 \pm 0.01$ & $30.31 \pm 0.12$ & $1.06 \pm 0.03$ & $0.23 \pm 0.01$ & $33.30 \pm 0.11$ & $0.77 \pm 0.02$ \\
\hline$\kappa 3-\mathrm{H} 1$ & $0.27 \pm 0.01$ & $35.41 \pm 0.15$ & $0.90 \pm 0.03$ & $0.31 \pm 0.01$ & $30.26 \pm 0.10$ & $0.92 \pm 0.02$ \\
\hline к3-Н2 & $0.32 \pm 0.01$ & $31.47 \pm 0.12$ & $0.98 \pm 0.03$ & $0.48 \pm 0.01$ & $32.91 \pm 0.13$ & $1.55 \pm 0.03$ \\
\hline$\kappa 3-\mathrm{H3}$ & $0.30 \pm 0.01$ & $31.52 \pm 0.12$ & $0.94 \pm 0.03$ & $0.24 \pm 0.01$ & $31.94 \pm 0.11$ & $0.78 \pm 0.02$ \\
\hline к3-H4 & $0.24 \pm 0.01$ & $35.67 \pm 0.17$ & $0.86 \pm 0.03$ & $0.36 \pm 0.01$ & $26.85 \pm 0.09$ & $0.97 \pm 0.02$ \\
\hline$\kappa 3-\mathrm{H5}$ & $0.23 \pm 0.01$ & $30.77 \pm 0.11$ & $0.72 \pm 0.03$ & $0.23 \pm 0.01$ & $35.48 \pm 0.13$ & $0.81 \pm 0.02$ \\
\hline к3-H6 & $0.32 \pm 0.01$ & $30.40 \pm 0.10$ & $0.95 \pm 0.02$ & $0.44 \pm 0.01$ & $32.45 \pm 0.16$ & $1.42 \pm 0.03$ \\
\hline к3-Н7 & $0.27 \pm 0.01$ & $29.36 \pm 0.11$ & $0.78 \pm 0.03$ & $0.29 \pm 0.01$ & $29.15 \pm 0.09$ & $0.86 \pm 0.02$ \\
\hline$\kappa 4-\mathrm{H} 1$ & $0.25 \pm 0.01$ & $30.68 \pm 0.11$ & $0.76 \pm 0.03$ & $0.36 \pm 0.01$ & $25.16 \pm 0.08$ & $0.89 \pm 0.02$ \\
\hline$\kappa 4-\mathrm{H} 2$ & $0.25 \pm 0.01$ & $32.17 \pm 0.12$ & $0.78 \pm 0.03$ & \multicolumn{3}{|c|}{ Not Produced } \\
\hline к4-H3 & $0.25 \pm 0.01$ & $30.90 \pm 0.12$ & $0.75 \pm 0.03$ & $0.23 \pm 0.01$ & $33.18 \pm 0.12$ & $0.75 \pm 0.02$ \\
\hline к4-H4 & $0.25 \pm 0.01$ & $34.25 \pm 0.14$ & $0.79 \pm 0.03$ & $0.25 \pm 0.01$ & $35.90 \pm 0.12$ & $0.89 \pm 0.02$ \\
\hline$\kappa 4-\mathrm{H5}$ & $0.32 \pm 0.01$ & $26.48 \pm 0.09$ & $0.83 \pm 0.02$ & $0.22 \pm 0.01$ & $34.78 \pm 0.12$ & $0.76 \pm 0.02$ \\
\hline$\kappa 4-\mathrm{H} 6$ & $0.27 \pm 0.01$ & $27.33 \pm 0.09$ & $0.75 \pm 0.02$ & $0.38 \pm 0.01$ & $23.19 \pm 0.08$ & $0.87 \pm 0.02$ \\
\hline$\kappa 4-\mathrm{H} 7$ & $0.28 \pm 0.01$ & $28.25 \pm 0.10$ & $0.81 \pm 0.02$ & $0.23 \pm 0.01$ & $37.89 \pm 0.14$ & $0.82 \pm 0.02$ \\
\hline к5-H1 & \multirow{2}{*}{\multicolumn{3}{|c|}{ Not Produced }} & \multirow{2}{*}{\multicolumn{3}{|c|}{ Not Produced }} \\
\hline$\kappa 5-\mathrm{H} 2$ & & & & & & \\
\hline$\kappa 5-\mathrm{H3}$ & $0.34 \pm 0.01$ & $28.58 \pm 0.10$ & $0.93 \pm 0.02$ & $0.32 \pm 0.01$ & $26.71 \pm 0.08$ & $0.84 \pm 0.02$ \\
\hline$\kappa 5-\mathrm{H} 4$ & \multirow{3}{*}{\multicolumn{3}{|c|}{ Not Produced }} & \multirow{4}{*}{\multicolumn{3}{|c|}{ Not Produced }} \\
\hline$\kappa 5-\mathrm{H} 5$ & & & & & & \\
\hline к5-Н6 & & & & & & \\
\hline к5-H7 & $0.32 \pm 0.01$ & $31.62 \pm 0.13$ & $1.01 \pm 0.03$ & & & \\
\hline к6-Н1 & $0.29 \pm 0.01$ & $30.14 \pm 0.10$ & $0.87 \pm 0.02$ & $0.32 \pm 0.01$ & $28.24 \pm 0.09$ & $0.89 \pm 0.02$ \\
\hline к6-H2 & $0.31 \pm 0.01$ & $31.57 \pm 0.11$ & $0.93 \pm 0.03$ & \multicolumn{3}{|c|}{ Not Produced } \\
\hline к6-Н3 & $0.28 \pm 0.01$ & $36.72 \pm 0.14$ & $1.00 \pm 0.03$ & $0.27 \pm 0.01$ & $30.80 \pm 0.11$ & $0.86 \pm 0.02$ \\
\hline к6-Н4 & $0.27 \pm 0.01$ & $37.20 \pm 0.15$ & $0.95 \pm 0.03$ & $0.36 \pm 0.01$ & $26.91 \pm 0.09$ & $0.97 \pm 0.02$ \\
\hline к6-H5 & $0.26 \pm 0.01$ & $33.74 \pm 0.13$ & $0.81 \pm 0.03$ & $0.22 \pm 0.01$ & $41.89 \pm 0.15$ & $0.90 \pm 0.03$ \\
\hline$\kappa 6-\mathrm{H6}$ & \multicolumn{3}{|c|}{ Not Produced } & $0.51 \pm 0.01$ & $36.25 \pm 0.22$ & $1.81 \pm 0.04$ \\
\hline к6-H7 & $0.28 \pm 0.01$ & $33.32 \pm 0.12$ & $0.89 \pm 0.03$ & $0.30 \pm 0.01$ & $31.67 \pm 0.12$ & $0.93 \pm 0.02$ \\
\hline
\end{tabular}

Figure 2. BLI measurements (KD, ka and kd) of Pertuzumab and Trastuzumab Vk1-6 and VH1-7 variants binding to the immobilised SpG biosensor. "Not Produced" denotes insufficient antibody production for the variants despite numerous large-scale transfections. All readings were obtained from at least three antibody concentrations. The readings were the average of independent triplicates. The graphs are shown in Supplementary Figures S5-S8. 


\subsection{BLI Measurement of Recombinant IgG1 Variants Binding to Protein L (PpL)}

Testing the same panel of antibodies on PpL (Figure 3), our systematic and holistic investigation of IgG1s to PpL showed non-canonical results of interactions with other Vk families and a contributory role of VH-FWR and CDRs to the interaction.

\begin{tabular}{|c|c|c|c|c|c|c|}
\hline \multirow{2}{*}{$\begin{array}{l}\text { Vк-VH } \\
\text { Pairing }\end{array}$} & \multicolumn{3}{|c|}{ Pertuzumab variants binding PpL } & \multicolumn{3}{|c|}{ Trastuzumab variants binding $P p L$} \\
\hline & $\begin{array}{c}\text { KD } \\
\left(M, 10^{-9}\right)\end{array}$ & $\begin{array}{c}\text { ka } \\
\left(1 / \mathrm{Ms}, 10^{4}\right)\end{array}$ & $\begin{array}{c}\text { kd } \\
\left(1 / \mathrm{s}, 10^{-4}\right)\end{array}$ & $\begin{array}{c}\text { KD } \\
\left(\mathrm{M}, 10^{-9}\right)\end{array}$ & $\begin{array}{c}\text { ka } \\
\left(1 / \mathrm{Ms}, 10^{4}\right)\end{array}$ & $\begin{array}{c}\text { kd } \\
\left(1 / \mathrm{s}, 10^{-4}\right)\end{array}$ \\
\hline к1-H1 & $0.76 \pm 0.01$ & $15.40 \pm 0.04$ & $1.15 \pm 0.02$ & \multirow{4}{*}{\multicolumn{3}{|c|}{ Not Produced }} \\
\hline$\kappa 1-\mathrm{H} 2$ & $0.68 \pm 0.01$ & $13.90 \pm 0.03$ & $0.93 \pm 0.02$ & & & \\
\hline к1-H3 & $0.53 \pm 0.01$ & $18.09 \pm 0.04$ & $0.84 \pm 0.02$ & & & \\
\hline$\kappa 1-\mathrm{H} 4$ & $0.57 \pm 0.01$ & $15.23 \pm 0.03$ & $0.83 \pm 0.02$ & & & \\
\hline к1-H5 & \multirow{2}{*}{\multicolumn{3}{|c|}{ Not Produced }} & $0.11 \pm 0.01$ & $15.71 \pm 0.02$ & $0.17 \pm 0.01$ \\
\hline$\kappa 1-\mathrm{H} 6$ & & & & \multicolumn{3}{|c|}{ Not Produced } \\
\hline к1-H7 & $0.68 \pm 0.01$ & $16.05 \pm 0.02$ & $1.08 \pm 0.01$ & $0.14 \pm 0.01$ & $11.02 \pm 0.01$ & $0.16 \pm 0.01$ \\
\hline$\kappa 2-\mathrm{H1}$ & $148.67 \pm 2.64^{*}$ & $6.36 \pm 0.11^{*}$ & $90.8 \pm 0.32^{*}$ & $124.41 \pm 52.86$ & $0.84 \pm 0.15$ & $9.49 \pm 0.15$ \\
\hline$\kappa 2-\mathrm{H} 2$ & $15.77 \pm 0.46^{+}$ & $1.68 \pm 0.03^{+}$ & $2.03 \pm 0.03^{+}$ & $78.63 \pm 7.94^{+}$ & $2.29 \pm 0.05^{+}$ & $7.71 \pm 0.05^{+}$ \\
\hline$\kappa 2-\mathrm{H} 3$ & $19.91 \pm 0.74$ & $0.57 \pm 0.02^{+}$ & $0.84 \pm 0.01^{+}$ & \multirow{3}{*}{\multicolumn{3}{|c|}{ Poor Response }} \\
\hline$\kappa 2-\mathrm{H} 4$ & $14.4 \pm 0.59$ & $0.36 \pm 0.01$ & $0.30 \pm 0.01$ & & & \\
\hline$\kappa 2-\mathrm{H} 5$ & $0.93 \pm 0.01$ & $8.72 \pm 0.01$ & $0.81 \pm 0.01$ & & & \\
\hline$\kappa 2-\mathrm{H6}$ & $0.72 \pm 0.01$ & $8.19 \pm 0.01$ & $0.59 \pm 0.01$ & $190.4 \pm 27.97^{+}$ & $0.24 \pm 0.02^{+}$ & $3.53 \pm 0.02^{+}$ \\
\hline$\kappa 2-\mathrm{H} 7$ & $46.40 \pm 1.07^{*}$ & $2.19 \pm 0.04^{*}$ & $9.66 \pm 0.05^{*}$ & $9.79 \pm 0.21^{+}$ & $1.51 \pm 0.04^{+}$ & $0.78 \pm 0.01^{+}$ \\
\hline$\kappa 3-\mathrm{H} 1$ & $38.03 \pm 1.73$ & $91.86 \pm 2.39$ & $86.08 \pm 1.21$ & $17.77 \pm 0.09$ & $11.68 \pm 0.05$ & $20.54 \pm 0.04$ \\
\hline$\kappa 3-\mathrm{H} 2$ & $25.42 \pm 0.30$ & $52.09 \pm 0.55$ & $127.73 \pm 0.57$ & $3.67 \pm 0.02$ & $7.63 \pm 0.02$ & $2.58 \pm 0.01$ \\
\hline$\kappa 3-\mathrm{H3}$ & $29.02 \pm 0.33$ & $48.16 \pm 0.50$ & $132.1 \pm 0.56$ & $4.34 \pm 0.04$ & $51.2 \pm 0.36$ & $21.33 \pm 0.07$ \\
\hline$\kappa 3-\mathrm{H} 4$ & $5.55 \pm 0.13$ & $53.95 \pm 0.61$ & $6.99 \pm 0.09$ & $5.50 \pm 0.04$ & $29.5 \pm 0.16$ & $15.52 \pm 0.05$ \\
\hline$\kappa 3-\mathrm{H} 5$ & $16.85 \pm 0.38$ & $72.65 \pm 1.41$ & $70.21 \pm 0.57$ & $4.54 \pm 0.03$ & $49.16 \pm 0.32$ & $22.25 \pm 0.07$ \\
\hline к3-Н6 & $23.21 \pm 0.35$ & $50.68 \pm 0.70$ & $85.00 \pm 0.44$ & $9.04 \pm 0.03$ & $15.37 \pm 0.04$ & $13.53 \pm 0.03$ \\
\hline$\kappa 3-\mathrm{H} 7$ & $17.66 \pm 0.33$ & $59.87 \pm 0.92$ & $74.33 \pm 0.48$ & $5.08 \pm 0.09$ & $46.18 \pm 0.71$ & $23.36 \pm 0.13$ \\
\hline$\kappa 4-\mathrm{H} 1$ & $74.56 \pm 1.25$ & $18.23 \pm 0.25$ & $123.00 \pm 0.62$ & $14.18 \pm 0.07$ & $9.15 \pm 0.04$ & $12.78 \pm 0.03$ \\
\hline$\kappa 4-\mathrm{H} 2$ & $37.05 \pm 0.50$ & $24.79 \pm 0.32$ & $87.04 \pm 042$ & \multicolumn{3}{|c|}{ Not Produced } \\
\hline к4-H3 & $13.09 \pm 0.20$ & $22.76 \pm 0.29$ & $19.45 \pm 0.13$ & $9.54 \pm 0.07$ & $17.19 \pm 0.09$ & $16.06 \pm 0.04$ \\
\hline$\kappa 4-\mathrm{H} 4$ & $13.66 \pm 0.24$ & $26.24 \pm 0.37$ & $20.37 \pm 0.14$ & $11.57 \pm 0.09$ & $16.95 \pm 0.12$ & $19.57 \pm 0.06$ \\
\hline$\kappa 4-\mathrm{H} 5$ & $54.38 \pm 0.42$ & $12.00 \pm 0.09$ & $64.34 \pm 0.15$ & $7.22 \pm 0.05$ & $20.05 \pm 0.12$ & $14.4 \pm 0.05$ \\
\hline$\kappa 4-\mathrm{H} 6$ & $23.49 \pm 0.50$ & $31.68 \pm 0.45$ & $47.04 \pm 0.29$ & $13.03 \pm 0.12$ & $14.32 \pm 0.11$ & $18.55 \pm 0.07$ \\
\hline$\kappa 4-\mathrm{H} 7$ & $34.95 \pm 0.39$ & $20.99 \pm 0.19$ & $65.46 \pm 0.23$ & $10.31 \pm 0.08$ & $20.34 \pm 0.12$ & $19.79 \pm 0.06$ \\
\hline$\kappa 5-\mathrm{H} 1$ & \multirow{2}{*}{\multicolumn{3}{|c|}{ Not Produced }} & \multirow{2}{*}{\multicolumn{3}{|c|}{ Not Produced }} \\
\hline$\kappa 5-\mathrm{H} 2$ & & & & & & \\
\hline к5-H3 & $13.58 \pm 0.05$ & $10.69 \pm 0.03$ & $13.93 \pm 0.03$ & $1.75 \pm 0.01$ & $10.80 \pm 0.01$ & $1.85 \pm 0.01$ \\
\hline$\kappa 5-\mathrm{H} 4$ & \multirow{3}{*}{\multicolumn{3}{|c|}{ Not Produced }} & \multirow{4}{*}{\multicolumn{3}{|c|}{ Not Produced }} \\
\hline$\kappa 5-\mathrm{H5}$ & & & & & & \\
\hline$\kappa 5-\mathrm{H6}$ & & & & & & \\
\hline к5-H7 & $13.88 \pm 0.13$ & $18.87 \pm 0.14$ & $23.50 \pm 0.08$ & & & \\
\hline$\kappa 6-\mathrm{H1}$ & \multirow{3}{*}{\multicolumn{3}{|c|}{ Poor Response }} & $14.93 \pm 0.23^{+}$ & $5.14 \pm 0.07^{+}$ & $7.53 \pm 0.06^{+}$ \\
\hline$\kappa 6-\mathrm{H} 2$ & & & & \multicolumn{3}{|c|}{ Not Produced } \\
\hline к6-Н3 & & & & $29.62 \pm 1.01^{*}$ & $6.07 \pm 0.07^{*}$ & $10.81 \pm 0.07^{*}$ \\
\hline$\kappa 6-\mathrm{H} 4$ & $43.46 \pm 1.76^{*}$ & $3.81 \pm 0.06^{*}$ & $10.43 \pm 0.07^{*}$ & $74.9 \pm 2.81^{*}$ & $1.65 \pm 0.02^{*}$ & $8.96 \pm 0.03^{*}$ \\
\hline к6-Н5 & $15.79 \pm 0.30^{+}$ & $1.77 \pm 0.03^{+}$ & $2.80 \pm 0.03^{+}$ & $14.84 \pm 0.65^{\circ}$ & $16.49 \pm 0.24^{*}$ & $11.17 \pm 0.14^{*}$ \\
\hline$\kappa 6-\mathrm{H6}$ & \multicolumn{3}{|c|}{ Not Produced } & $82.16 \pm 2.13^{*}$ & $1.28 \pm 0.02^{*}$ & $8.36 \pm 0.03^{*}$ \\
\hline$\kappa 6-\mathrm{H} 7$ & $40.69 \pm 2.57$ & $0.31 \pm 0.01$ & $0.80 \pm 0.01$ & $60.37 \pm 0.50$ & $1.77 \pm 0.01$ & $9.83 \pm 0.01$ \\
\hline
\end{tabular}

Figure 3. BLI measurements (KD, ka, and kd) of Pertuzumab and Trastuzumab Vk1-6 and VH1-7 variants binding to the immobilised PpL biosensor. "Not Produced" denotes insufficient antibody production for the variants despite numerous large-scale transfections. "Poor response" indicates that the particular $\mathrm{V} \kappa-\mathrm{VH}$ IgG1 pairing was unable to give response rates within the detection limit across all concentrations. * denotes readings that were derived from two IgG1 concentrations. + denotes represent readings derived from only one IgG1 concentration which was deemed reliable. All other readings were obtained from at least three antibody concentrations. The readings were the average of independent triplicates. The graphs are shown in Supplementary Figures S9-S12.

As a control for expected superantigen interactions, the Pertuzumab IgG1s of Vk1, 3, and 4 interacted with PpL, where Vk1 showed the lowest KD range $\left(0.53-0.76 \times 10^{-9} \mathrm{M}\right)$, 
followed by Vк3 $\left(5.55-38.03 \times 10^{-9} \mathrm{M}\right)$ and $\mathrm{V}_{\kappa} 4\left(13.09-74.56 \times 10^{-9} \mathrm{M}\right)$ (refer to Supplementary Figures S1-S4 for the graphs). The $V_{k} 1$ findings were consistent with our previous work $[8,28,29]$. The lower $K D$ s of the $V \kappa 3$ and 4 were due to the lower dissociation rates $(\mathrm{kd})$ despite the higher association rates $(\mathrm{ka})$ than $\mathrm{V}_{k} 1$. This trend was also observed for the Trastuzumab IgG1s with its $\mathrm{VK}_{\mathrm{K}}$ showing the lowest $\mathrm{KD}$ range $\left(0.11\right.$ and $\left.0.14 \times 10^{-9} \mathrm{M}\right)$ followed by Vk3 $\left(3.67-17.77 \times 10^{-9} \mathrm{M}\right)$ and $4\left(7.22-14.18 \times 10^{-9} \mathrm{M}\right)$. Note that Trastuzumab IgG1s showed lower and a narrower KD range than the Pertuzumab Vk-VH equivalents suggesting effects from the CDRs which were what differed between the two sets of IgG1s.

Unexpectedly, certain Pertuzumab VHs paired with Vk2, 5 and 6 exhibited interactions with PpL. Amongst these Vk families, Pertuzumab variants Vk5 $\left(13.58 \& 13.88 \times 10^{-9} \mathrm{M}\right)$, $6\left(15.79-43.46 \times 10^{-9} \mathrm{M}\right)$ and certain Vk2 variants $\left(\mathrm{VH} 2-4\right.$ and $\left.7,14.4-46.4 \times 10^{-9} \mathrm{M}\right)$ had KDs comparable to Vk3 and 4 variants $\left(5.55-74.56 \times 10^{-9} \mathrm{M}\right)$. Pertuzumab Vk2 paired with VH5 and 6 ( 0.93 and $0.72 \times 10^{-9} \mathrm{M}$, respectively) had KDs comparable to Vk1 $\left(0.53-0.76 \times 10^{-9} \mathrm{M}\right)$ while VK2 paired with VH1 had the highest KD (poorest binding) of $148.67 \times 10^{-9} \mathrm{M}$. There were also non-binding IgG1s of the Pertuzumab Vk6 variant with VH1-3 (Poor Response) despite measurable responses when paired with VH4, 5 and 7.

Generally, the Pertuzumab trends were largely similar to the Trastuzumab IgG1s where KDs of Vk5 $\left(1.75 \times 10^{-9} \mathrm{M}\right)$ and $6\left(14.84-82.16 \times 10^{-9} \mathrm{M}\right)$ and $\mathrm{V} \kappa 2$ paired with VH2 \& 7 (78.63 \& $9.79 \times 10^{-9} \mathrm{M}$, respectively) had $\mathrm{KD}$ values comparable to VK3 and $4\left(3.67-14.18 \times 10^{-9} \mathrm{M}\right)$. Trastuzumab Vk2 paired with VH1 and 6 had the highest KD (poorest interaction) at $124.41 \& 190.4 \times 10^{-9} \mathrm{M}$, respectively. The non-binders were Trastuzumab Vk2 paired with VH3-5 (Poor Response) rather than in the Vk6 family observed for Pertuzumab. These differences demonstrated a role of the CDRs and their contribution to the PpL engagement.

\section{Discussion}

We set out to investigate the interactions of antibody superantigens Protein A, G, and $\mathrm{L}$ systematically and holistically with the engineering of the various V-regions of IgG1 antibodies. By using CDRs of Pertuzumab and Trastuzumab grafted onto Vk1-6 and VH1-7 FWRs, measurements to the superantigens showed no major differences for SpA between Pertuzumab and Trastuzumab IgG1 variants (Figure 1). This was expected given that SpA bound IgG1s predominantly at the $\mathrm{CH} 2-\mathrm{CH} 3$ domains [30] with some contributions from the VH3 framework [4] that is also observed here to a lesser extent where the VK chains paired with VH3 showed a slightly lower KD (better) measurement compared to the other variants. Yet, this difference is notably less pronounced compared to our previous work on IgEs with the same Vks-VH [4], where the VH3-CDR2 S58 residue had a more significant role in SpA binding for IgEs possibly due to its structural contribution and the $\mathrm{C} \varepsilon$ region.

With respect to SpG interactions, no notable differences in KDs (Figure 2) were observed among the 63 IgG1 variants. Admittedly, there are other non-specific purification methods such as the Melon ${ }^{\mathrm{TM}}$ gel which could avoid biases introduced by Protein $\mathrm{G}$ affinity purification. However, given that our culture medium contains low Ig FBS, utilising such a method could result in potential cross contamination of unspecific bovine antibodies (such as IgA and IgM, even with an additional size exclusion step). We thus utilised Protein G purification for consistency with our previous work [21] in which we performed similar analyses. Additionally, SpA interactions were found to be highly consistent (Figure 1) despite binding to a different site from SpG [31], reaffirming that there is no rationale to suspect interferences from using SpG purification on the Octet ${ }^{\circledR}$ measurements. Nonetheless, future studies can utilise other immunoaffinity resins or the Melon gel if they wish to confirm these findings. Notably, our size exclusion purification of monomeric IgG fractions does not show residual binding of SpG to interfere with our subsequent measurements and that affinity purifications do not rely on the small changes in $\mathrm{ka}$ and $\mathrm{kd}$ that are measured here, but on the general binding between the antibody and SpG. In fact, we found a narrow $\mathrm{KD}$ range between the IgGs and SpG that is likely due to the lack of interference from the 
V-regions present for SpA. While SpG was previously reported [32] to bind to IgG1 better than SpA, this trend was more pronounced in our study.

Measurements of PpL interactions to Pertuzumab and Trastuzumab variants expectedly showed that $\mathrm{VHs}$ paired with $\mathrm{V} \kappa 1,3$ and 4 exhibits interactions as previously reported $[7,8]$. Surprisingly, we found non-canonical interaction of PpL with Vк2 previously determined to not bind PpL [7] while there were no known reports of $\mathrm{V} \kappa 5$ \& 6 interactions at the time of writing. Producing these light chains as secreted dimers, we also affirmed that Vk2, 5 and 6 light chains did not interact with PpL on the same BLI experiments (Supplementary Figure S13). Notable binders to PpL are: Pertuzumab Vк2-VH1-7; Vk5-VH3 \& 7; Vк6-VH4, 5 \& 6; Trastuzumab Vк2-VH1, 2, 5 \& 6; Vк5-VH3; Vк6-VH1, $3-7$ (Figure 3).

Although the novel Vk IgG1s bound PpL showed comparable KDs, it should be noted that the KDs were calculated from one (denoted as " + " in Figure 3) or two (denoted as "**) antibody concentrations, generally from the highest concentrations (100 $\mathrm{nM}$ and below) of the Ig variant. The notable exceptions were that of Pertuzumab Vk2-VH4-6, Vk5-VH3 \& 7,

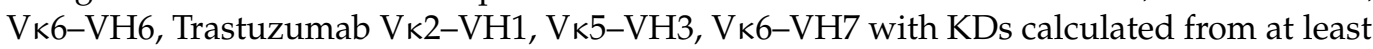
three concentrations. Interestingly, two variants: Pertuzumab Vк2-VH5 \& 6 showed KDs comparable to $\mathrm{V} \kappa 1-\mathrm{VH}$ s values.

The unexpected IgG1 variants interacting with PpL suggested a combined VH-Vk induced binding site to PpL that may be similar to the non-canonical binding of IgEs to nickel [4] in our previous work using the same V-regions. In fact, the IgG1 variants were validated with the expected interactions to SpA and SpG in this study to complement our previous work on their interactions with Fc $\gamma 2 \mathrm{~A}$ and Her2 [21]. Given the lack of interactions between $V_{\kappa} 2,5$, and 6 with PpL and consistency between the Trastuzumab and Pertuzumab variants, where Pertuzumab Vk6-VH1-3 and Trastuzumab Vk2-VH3-5 were non-binders (labelled as "Poor Response" pairs in Figure 3), PpL interaction involved more than V-region pairings alone.

With the differences between the Pertuzumab and Trastuzumab which share very similar V-regions, our findings further demonstrate the need for a design thinking [12] approach involving holistic antibody investigations approach [20]. Such an approach allowed detailed investigations for unexpected interactions between the antibodies with other proteins that can have notable immune effects, as was with our unexpected findings of IgAs binding to SpG [9]. With relevance to the development of therapeutics where a personalised antibody approach may be beneficial to avoid unwanted side effects, such interactions may also be engineered for purification purposes.

Supplementary Materials: The following supporting information can be downloaded at: https:/ / www.mdpi.com/article/10.3390/antib11010014/s1, Figure S1: BLI measurement of Pertuzumab and Trastuzumab Vk1-6 binding to immobilised PpL biosensor, Figure S2: Binding graph of Pertuzumab variants (PVK3 PVH7 to PVK6 PVH7) to Protein A, Figure S3: Binding graph of Trastuzumab variants (HVK1 HVH5 to HVK3 HVH5) to Protein A, Figure S4: Binding graph of Trastuzumab variants (HVK3 HVH7 to HVK6 HVH7) to Protein A, Figure S5: Binding graph of Pertuzumab variants (PVK1 PVH1 to PVK3 PVH5) to Protein G, Figure S6: Binding graph of Pertuzumab variants (PVK3 PVH7 to PVK6 PVH7) to Protein G, Figure S7: Binding graph of Trastuzumab variants (HVK1 HVH5 to HVK3 HVH5) to Protein G, Figure S8: Binding graph of Trastuzumab variants (HVK3 HVH7 to HVK6 HVH7) to Protein G, Figure S9: Binding graph of Pertuzumab variants (PVK1 PVH1 to PVK3 PVH5) to Protein L, Figure S10: Binding graph of Pertuzumab variants (PVK3 PVH7 to PVK6 PVH7) to Protein L, Figure S11: Binding graph of Trastuzumab variants (HVK1 HVH5 to HVK3 HVH5) to Protein L, Figure S12: Binding graph of Trastuzumab variants (HVK3 HVH7 to HVK6 HVH7) to Protein L, Figure S13: BLI measurement of Pertuzumab and Trastuzumab Vk1-6 binding to immobilized PpL biosensor.

Author Contributions: Conceptualisation, W.-L.L. and S.K.-E.G.; methodology, W.-L.L. and S.K.E.G.; validation, J.Y.Y.; formal analysis, W.-L.L. and S.K.-E.G.; investigation, W.-L.L. and S.K.-E.G.; writing-original draft preparation, W.-L.L. and S.K.-E.G.; writing-review and editing, W.-L.L., Y.-L.N. and S.K.-E.G.; supervision, S.K.-E.G., Y.-L.N. and A.W.; funding acquisition, S.K.-E.G. All authors have read and agreed to the published version of the manuscript. 
Funding: This research was partially supported by the Joint Council Office, Agency for Science, Technology, and Research, Singapore under Grant number JCO1334i00050 and the National Research Foundation (NRF) Singapore grant to Experimental Drug Development Centre (EDDC).

Institutional Review Board Statement: Not applicable.

Informed Consent Statement: Not applicable.

Data Availability Statement: Data is available upon request.

Conflicts of Interest: The authors declare no conflict of interest. The funders had no role in the design of the study; in the collection, analyses, or interpretation of data; in the writing of the manuscript; or in the decision to publish the results.

\section{References}

1. Deacy, A.M.; Gan, S.K.-E.; Derrick, J.P. Superantigen Recognition and Interactions: Functions, Mechanisms and Applications. Front. Immunol. 2021, 12, 731845. [CrossRef] [PubMed]

2. Spaulding, A.R.; Salgado-Pabón, W.; Kohler, P.L.; Horswill, A.R.; Leung, D.Y.M.; Schlievert, P.M. Staphylococcal and Streptococcal Superantigen Exotoxins. Clin. Microbiol. Rev. 2013, 26, 422-447. [CrossRef] [PubMed]

3. Sjöbring, U.; Björck, L.; Kastern, W. Streptococcal protein G. Gene structure and protein binding properties. J. Biol. Chem. 1991, 266, 399-405. [CrossRef]

4. Su, C.T.-T.; Lua, W.-H.; Poh, J.-J.; Ling, W.-L.; Yeo, J.Y.; Gan, S.K.-E. Molecular Insights of Nickel Binding to Therapeutic Antibodies as a Possible New Antibody Superantigen. Front. Immunol. 2021, 12, 676048. [CrossRef]

5. Grov, A.; Myklestad, B.; Oeding, P. Immunochemical Studies on Antigen Preparations from Staphylococcus Aureus. Acta Pathol. Microbiol. Scand. 1964, 61, 588-596. [CrossRef]

6. Sasso, E.H.; Silverman, G.J.; Mannik, M. Human IgA and IgG F(ab')2 that bind to staphylococcal protein A belong to the VHIII subgroup. J. Immunol. 1991, 147, 1877-1883.

7. Nilson, B.H.; Solomon, A.; Björck, L.; Akerström, B. Protein L from Peptostreptococcus magnus binds to the kappa light chain variable domain. J. Biol. Chem. 1992, 267, 2234-2239. [CrossRef]

8. Su, C.T.-T.; Ling, W.-L.; Lua, W.-H.; Poh, J.-J.; Gan, S.K.-E. The role of Antibody Vk Framework 3 region towards Antigen binding: Effects on recombinant production and Protein L binding. Sci. Rep. 2017, 7, 3766. [CrossRef]

9. Ling, W.-L.; Su, C.T.-T.; Lua, W.-H.; Yeo, J.Y.; Poh, J.-J.; Ng, Y.-L.; Wipat, A.; Gan, S.K.-E. Engaging the 'A' Class Antibody: Variable-Heavy $(\mathrm{VH})$ region influencing IgA1\&2 engagement of Fc $\alpha$ RI and superantigen proteins $\mathrm{G}, \mathrm{A}$, and L. bioRxiv 2021. [CrossRef]

10. Samsudin, F.; Yeo, J.Y.; Gan, S.K.-E.; Bond, P.J. Not all therapeutic antibody isotypes are equal: The case of IgM versus IgG in Pertuzumab and Trastuzumab. Chem. Sci. 2020, 11, 2843-2854. [CrossRef]

11. Gan, S.K.-E.; Yeo, J.Y. Sagacious Epitope Selection for Vaccines, and Both Antibody-Based Therapeutics and Diagnostics: Tips From Virology and Oncology. Preprints 2021. [CrossRef]

12. Ling, W.-L.; Lua, W.-H.; Gan, S.K.-E. Sagacity in antibody humanization for therapeutics, diagnostics and research purposes: Considerations of antibody elements and their roles. Antib. Ther. 2020, 3, 71-79. [CrossRef] [PubMed]

13. Janeway, C.A.; Travers, P.; Walport, M.; Capra, D.J. Immunobiology; Taylor \& Francis Group/Garland Science: London, UK, 2001.

14. Haraldsson, Á.; Kock-Jansen, M.J.H.; Jaminon, M.; v Eck-Arts, P.B.J.M.; de Boo, T.; Weemaes, C.M.R.; Bakkeren, J.A.J.M. Determination of Kappa and Lambda Light Chains in Serum Immunoglobulins G, A and M. Ann. Clin. Biochem. 1991, 28, 461-466. [CrossRef] [PubMed]

15. Alroy, I.; Yarden, Y. The ErbB signaling network in embryogenesis and oncogenesis: Signal diversification through combinatorial ligand-receptor interactions. FEBS Lett. 1997, 410, 83-86. [CrossRef]

16. Olayioye, M.A.; Neve, R.M.; Lane, H.A.; Hynes, N.E. The ErbB signaling network: Receptor heterodimerization in development and cancer. Embo J. 2000, 19, 3159-3167. [CrossRef] [PubMed]

17. Carter, P.; Presta, L.; Gorman, C.M.; Ridgway, J.B.; Henner, D.; Wong, W.L.; Rowland, A.M.; Kotts, C.; Carver, M.E.; Shepard, H.M. Humanization of an anti-p185HER2 antibody for human cancer therapy. Proc. Natl. Acad. Sci. USA 1992, 89, 4285-4289. [CrossRef] [PubMed]

18. Fendly, B.M.; Winget, M.; Hudziak, R.M.; Lipari, M.T.; Napier, M.A.; Ullrich, A. Characterization of murine monoclonal antibodies reactive to either the human epidermal growth factor receptor or HER2/neu gene product. Cancer Res. 1990, 50, 1550-1558.

19. Lua, W.-H.; Gan, S.K.-E.; Lane, D.P.; Verma, C.S. A search for synergy in the binding kinetics of Trastuzumab and Pertuzumab whole and $\mathrm{F}(\mathrm{ab})$ to Her2. NPJ Breast Cancer 2015, 1, 15012. [CrossRef]

20. Phua, S.-X.; Chan, K.-F.; Su, C.T.-T.; Poh, J.-J.; Gan, S.K.-E. Perspective: The promises of a holistic view of proteins-impact on antibody engineering and drug discovery. Biosci. Rep. 2019, 39, BSR20181958. [CrossRef]

21. Ling, W.-L.; Lua, W.-H.; Poh, J.-J.; Yeo, J.Y.; Lane, D.P.; Gan, S.K.-E. Effect of VH-VL Families in Pertuzumab and Trastuzumab Recombinant Production, Her2 and Fc $\gamma$ IIA Binding. Front. Immunol. 2018, 9, 469. [CrossRef] 
22. Ling, W.-L.; Su, C.T.-T.; Lua, W.-H.; Poh, J.-J.; Ng, Y.-L.; Wipat, A.; Gan, S.K.-E. Essentially Leading Antibody Production: An Investigation of Amino Acids, Myeloma, and Natural V-Region Signal Peptides in Producing Pertuzumab and Trastuzumab Variants. Front. Immunol. 2020, 11, 604318. [CrossRef] [PubMed]

23. Lua, W.-H.; Ling, W.-L.; Yeo, J.Y.; Poh, J.-J.; Lane, D.P.; Gan, S.K.-E. The effects of Antibody Engineering CH and CL in Trastuzumab and Pertuzumab recombinant models: Impact on antibody production and antigen-binding. Sci. Rep. 2018, 8, 718. [CrossRef] [PubMed]

24. Lua, W.-H.; Su, C.T.-T.; Yeo, J.Y.; Poh, J.-J.; Ling, W.-L.; Phua, S.-X.; Gan, S.K.-E. Role of the IgE variable heavy chain in FceRI $\alpha$ and superantigen binding in allergy and immunotherapy. J. Allergy Clin. Immunol. 2019, 144, 514-523.e515. [CrossRef] [PubMed]

25. Chan, W.-T.; Verma, C.S.; Lane, D.P.; Gan, S.K.-E. A comparison and optimization of methods and factors affecting the transformation of Escherichia coli. Biosci. Rep. 2013, 33, e00086. [CrossRef]

26. Heng, Z.S.-L.; Yeo, J.Y.; Koh, D.W.-S.; Gan, S.K.-E.; Ling, W.-L. Augmenting recombinant antibody production in HEK293E cells: Optimising transfection and culture parameters. Antib. Ther. 2022, 5, 30-41. [CrossRef]

27. Su, C.T.-T.; Lua, W.-H.; Ling, W.-L.; Gan, S.K.-E. Allosteric Effects between the Antibody Constant and Variable Regions: A Study of IgA Fc Mutations on Antigen Binding. Antibodies 2018, 7, 20. [CrossRef]

28. Rodrigo, G.; Gruvegård, M.; Van Alstine, J.M. Antibody Fragments and Their Purification by Protein L Affinity Chromatography. Antibodies 2015, 4, 259-277. [CrossRef]

29. Åkerström, B.; Björck, L. Protein L: An Immunoglobulin Light Chain-binding Bacterial Protein: Characterization of binding and physicochemical properties. J. Biol. Chem. 1989, 264, 19740-19746. [CrossRef]

30. Deisenhofer, J. Crystallographic refinement and atomic models of a human Fc fragment and its complex with fragment B of protein A from Staphylococcus aureus at 2.9- and 2.8-. ANG resolution. Biochemistry 1981, 20, 2361-2370. [CrossRef]

31. Kato, K.; Lian, L.-Y.; Barsukov, I.L.; Derrick, J.P.; Kim, H.; Tanaka, R.; Yoshino, A.; Shiraishi, M.; Shimada, I.; Arata, Y.; et al. Model for the complex between protein $\mathrm{G}$ and an antibody FC fragment in solution. Structure 1995, 3, 79-85. [CrossRef]

32. Choe, W.; Durgannavar, T.A.; Chung, S.J. Fc-Binding Ligands of Immunoglobulin G: An Overview of High Affinity Proteins and Peptides. Materials 2016, 9, 994. [CrossRef] [PubMed] 